\title{
The quantum world is not built up from correlations
}

Found. Phys. 36, 1573-1586 (2006).

\author{
Michael Seevinck \\ Institute of History and Foundations of Science, Utrecht University, \\ P.O Box 80.000, 3508 TA Utrecht, The Netherlands. \\ E-mail: seevinck@phys.uu.nl
}

It is known that the global state of a composite quantum system can be completely determined by specifying correlations between measurements performed on subsystems only. Despite the fact that the quantum correlations thus suffice to reconstruct the quantum state, we show, using a Bell inequality argument, that they cannot be regarded as objective local properties of the composite system in question. It is well known since the work of J.S. Bell, that one cannot have locally preexistent values for all physical quantities, whether they are deterministic or stochastic. The Bell inequality argument we present here shows this is also impossible for correlations among subsystems of an individual isolated composite system. Neither of them can be used to build up a world consisting of some local realistic structure. As a corrolary to the result we argue that entanglement cannot be considered ontologically robust. The argument has an important advantage over others because it does not need perfect correlations but only statistical correlations. It can therefore easily be tested in currently feasible experiments using four particle entanglement.

Keywords: ontology, quantum correlations, Bell inequality, entanglement.

\section{INTRODUCTION}

What is quantum mechanics about? This question has haunted the physics community ever since the conception of the theory in the 1920's. Since the work of John Bell we know at least that quantum mechanics is not about a local realistic structure built up out of values of physical quantities [1. This is because of the well known fact that if one considers the values of physical quantities to be locally real, they must obey a so-called Bell inequality, which quantum mechanics violates. The paradigmatic example of a quantum system giving rise to such a violation is the singlet state of two spin- $\frac{1}{2}$ particles. This state describes two par- 
ticles that are anti-correlated in spin. Bell's result shows that no single particle in the singlet state can be regarded to have a locally preexistent spin value. Instead, the singlet state tells us that upon measurement the spin values, if measured in the same direction on each particle, will always be found anti-parallel. Because this (anti-) correlation is found in all such measurements, an obvious question to ask is whether or not we can think of this (anti-) correlation as a real property of the two particle system independent of measurement.

Could it be that what is real about two systems in the singlet state are not the local spin values, but merely the correlations between the two systems? Is quantum mechanics about a world consisting not of objective values of quantities but solely of objective correlations, of which some are revealed in experiment? In other words, is there a fundamental difference according to quantum mechanics as regards the physical status of values of quantities and of correlations, as for example Mermin [2] seems to suggest?

There is good reason to think that these questions should be answered in the positive, since a non-trivial theorem (which is true in quantum mechanics) points into this direction. The theorem (to be treated in the next section) shows that the global state of a composite quantum system can be completely determined by specifying correlations (joint probability distributions) when sufficient local measurements are performed on each subsystem. It thus suffices to consider only correlations when completely specifying the state of the composite system. But can one also think of these correlations to be objective properties that pertain to the composite quantum system in question? As mentioned already in the case of the anti-correlation of the singlet state, one is tempted to think that this is indeed the case. However in this letter we will demonstrate that, however tempting, no such interpretation is possible and that these questions (as well as the questions mentioned earlier) can thus not be answered in the positive.

Cabello [3] and Jordan [4] give the same answer to similar questions using a Kochen-Specker [5], Greenberger-Horne-Zeilinger (GHZ) [6] or Hardy [7] argument. Besides giving the Bell inequality version of the argument (which in a sense completes the discussion because it was still lacking), the advantage of the argument given here above these previous arguments, is that it is more easily experimentally accessible using current technology. For this purpose, we explicitly present a quantum state and the measurements that are to be performed in order to test the inequality.

The structure of this paper is as follows. In sec. 2 we will present an argument to the effect that quantum correlations are real objective properties pertaining to composite quantum systems. In the next two sections we will however show that this line of thought is in conflict with quantum mechanics itself. To get such a conclusive result we need to be very formal and rigorous. In sec. 3 we will therefore define our notion of correlation and derive a Bell inequality for correlations using a stochastic hidden variable model under the assumption of local realism, which formalises the idea of correlations as objective local properties. In sec. 4 we show that this inequality, when turned into it's quantum mechanical form, is violated by quantum correlations. We present a quantum state and a set of measurements that allow for such a violation and furthermore show that it is the maximum possible violation. In sec. 5 we apply this result to show that 
entanglement cannot be considered ontologically robust when the quantum state is taken to be a complete description. However, we argue that it nevertheless can be considered a resource in quantum information theory to perform computational and information-theoretic tasks. In the last section, sec. 6, we briefly discuss the implications of our results, compare our argument to the ones given by Cabello [3] and by Jordan [4] and return to the questions stated in the beginning of this introduction.

\section{DOES THE QUANTUM WORLD CONSIST OF COR- RELATIONS?}

In many important instances a system can be regarded as composed out of separate subsystems. In a physical theory that describes such composite systems it can be asked whether one can assume that the global state of the system can be completely determined by specifying correlations (joint probability distributions) when a sufficient number of local measurements are performed on each subsystem (note that here (and in the rest of the paper) 'local' is taken to be opposed to 'global' and thus not in the sense of spatial localisation(1). Barrett [8] calls this the global state assumption. Perhaps not surprisingly, the assumption holds for classical probability theory and for quantum mechanics on a complex Hilbert space. However, it need not be satisfied in an arbitrary theory, which shows that the theorem is non-trivial. For example, Wootters [9] has shown that for quantum mechanics on a real Hilbert space the assumption does not hold because the correlations between subsystems do not suffice to build up the total state. By counting available degrees of freedom of the state of a composite system and of the states of its subsystems one can easily convince oneself that this is the case 2 .

Mermin has called the fact that in quantum mechanics the global state assumpion holds sufficiency of subsystems correlations, or the SSC theorem [10]. He phrases it as follows. Given a system $\mathcal{S}=\mathcal{S}_{1}+\mathcal{S}_{2}$ with density matrix $W$, then $W$ is completely determined by correlations (joint probability distributions) $\operatorname{Tr}\left[W\left(A_{i} \otimes B_{j}\right)\right]$ for an appropriate set of observable pairs $\left\{A_{i}\right\},\left\{B_{j}\right\}$, where $A_{i}=A_{i} \otimes \mathbb{1}$ is an observable for subsystem $\mathcal{S}_{1}$ and $B_{j}=\mathbb{1} \otimes B_{j}$ is an observable for subsystem $\mathcal{S}_{2}$. The proof 3 relies on three facts: Firstly, the mean values of all observables for the entire system determine its state. Secondly, the set of all products over subsystems of subsystem observables (i.e., the set $\left\{A_{i} \otimes B_{j}\right\}$ ) contains a basis for the algebra of all such system-wide observables. Thirdly, the algorithm that supplies observables with their mean value is linear on the algebra of observables.

As an example of the theorem, consider the well known singlet state written

\footnotetext{
${ }^{1}$ Local thus refers to being confined to a subsystem of a larger system, without requiring the subsystem itself to be localised (it can thus itself exist of spatially separated parts).

${ }^{2} \mathrm{~J}$. Barrett (private communication) gives the following counting argument. A density matrix on a real Hilbert space with dimension $d$ has $N=\left(d^{2}-d\right) / 2+d=(1 / 2) d(d+1)$ parameters (without normalization), and a density matrix on a $d \otimes d$-dimensional Hilbert space has $(1 / 2) d^{2}\left(d^{2}+1\right)$ parameters, which is too many because it is more than $N^{2}$.

${ }^{3}$ Wootters has also independently proven this, see 9 .
} 
as the one-dimensional projection operator

$$
\hat{P}_{\text {singlet }}=\frac{1}{4}\left(\mathbb{1}-\sigma_{z}^{1} \otimes \sigma_{z}^{2}-\sigma_{x}^{1} \otimes \sigma_{x}^{2}-\sigma_{y}^{1} \otimes \sigma_{y}^{2}\right) .
$$

The mean value of $\hat{P}_{\text {singlet }}$ is determined by the mean values of the products of the $x, y$ and $z$ components of the individual spins. Since the mean value of this projector is 1 for the singlet, the singlet state is thus determined by the spin correlations in $x, y$ and $z$ direction having the value -1 (perfect anti-correlation). Perfect anti-correlation of any three orthogonal components is thus enough to ensure that the global state is the singlet state. Thus correlations among all subsystems completely determine the density matrix for the composite system they make up, or in Mermin's words [10]: "anything you can say in terms of quantum states can be translated into a statement about subsystem correlations, i.e., about joint distributions."

It is tempting to think that because of this theorem and because of the fact that Bell has shown that a quantum state is not a prescription of local realistic values of physical quantities, that we can take a quantum state to be nothing but the encapsulation of all the quantum correlations present in the quantum system. Indeed, the SSC theorem was used by N. David Mermin in 1998 to argue for the idea that correlations are physically real whereas values of quantities are not [2] (although by now he has set these ideas asidet). Without wanting to claim that Mermin is committed to the issue we address next, we explore if correlations between subsystems of an individual isolated composed system, although determining the state of the total composite system, can also be be considered to be real objective local properties of such a system. That is, can one consider quantum correlations to be local realistic properties that somehow (pre)exist in the quantum state? Are correlations somehow atomic building blocks of the (quantum) world?

In the next two sections we will show that none of these questions can be answered in the positive. We will be formal and rigorous and follow the road paved by John Bell for us, but enlargen it to not only include values of quantities but also correlations.

\section{A BELL INEQUALITY FOR CORRELATIONS}

Consider two spatially separated parties $I$ and $I I$ which each have a bi-partite system. Furthermore, assume that each party determines the correlations of the bi-partite system at his side. By correlations we here mean the joint probability distributions $P_{A B}^{I}(a b)$ and $P_{C D}^{I I}(c d)$, where $A$ and $B$ are physical quantities each associated to one of the subsystems in the bi-partite system that party $I$ has, and where $a$ and $b$ denote the possible values these quantities can obtain. The same holds for quantities $C, D$ and possible outcomes $c, d$ but then for party $I I$. We now assume local realism for these correlations in the following well-known way. Firstly, the correlations party $I$ finds are determined by some hidden variable $\lambda \in \Lambda$ (with distribution $\rho(\lambda)$ and hidden variable space $\Lambda$ ). The same of course

\footnotetext{
${ }^{4}$ N.D. Mermin, personal communication.
} 
holds for $I I$. Secondly, because of locality the correlations one party will obtain are for a given $\lambda$ statistically independent of the correlations that the other party will find 5 . Under these assumptions the joint probability distribution over the four possible outcomes factorises which gives:

$$
P_{A B, C D}(a b, c d)=\int_{\Lambda} P_{A B}^{I}(a b \mid \lambda) P_{C D}^{I I}(c d \mid \lambda) \rho(\lambda) d \lambda .
$$

Here we assume a so-called stochastic hidden variables model where the hidden variables $\lambda$ determine only the joint probabilities $P_{A B}^{I}(a b \mid \lambda), P_{C D}^{I I}(c d \mid \lambda)$ (i.e., correlations), and not the values of the quantities themselves. Furthermore, the joint probability $P_{A B}(a b \mid \lambda)$ itself need not factorise (the same of course holds for $P_{C D}(c d \mid \lambda)$.

Suppose now that we deal with dichotomic quantities $A, B, C, D$ with possible outcomes $a, b, c, d \in\{-1,1\}$. The mean value of the product of two correlations is given by

$$
E(A B, C D)=\sum_{a, b, c, d} a b c d P_{A B, C D}(a b, c d)
$$

Then because of the factorisability of Eq.(2) we get the following Bell inequality, in so-called CHSH form [11,

$$
\begin{aligned}
& \mid E(A B, C D)+E\left(A B,(C D)^{\prime}\right) \\
& \quad+E\left((A B)^{\prime}, C D\right)-E\left((A B)^{\prime},(C D)^{\prime}\right) \mid \leq 2 .
\end{aligned}
$$

Here $A B,(A B)^{\prime}$ denote two sets of quantities that give rise to two different joint probabilities (i.e., correlations) at party $I$. Similarly for the set $C D$ and $(C D)^{\prime}$ at party $I I$.

This is the Bell inequality in terms of correlations that will be used in the next section. Despite the resemblance between our inequality and the usual CHSH inequality, they are fundamentally different because the latter is in terms of subsystems quantities whereas the former is in terms of correlations and does not assume anything about the values of subsystems quantities, which the usual inequality does.

\section{QUANTUM CORRELATIONS ARE NOT LOCAL EL- EMENTS OF REALITY}

Consider a four-partite quantum system $\mathfrak{O}$ that consists of two pairs of spin$\frac{1}{2}$ particles where parties $I$ and $I I$ each receive a single pair. In this section

${ }^{5}$ To be more specific, the doctrine of local realism leading to the Bell-inequalities is the conjunction of the following three assumptions. (i) Outcome independence. The probability to obtain results for the observables measured on one side is completely determined by the experimental setup and the hidden variable $\lambda$. There is no dependency on the results obtained on the other side. (ii) Parameter independence. The probability to obtain results is only locally determined, i.e., it is independent of the distant measurement devices. (iii) Autonomy of the source. The hidden variable distribution $\rho(\lambda)$ of the source is independent of the particularly chosen measurement setup. The conjunction of these three lead to the result that the joint probability distribution $P_{A B, C D}(a b, c d \mid \lambda)$ factorises: $P_{A B, C D}(a b, c d \mid \lambda)=P_{A B}^{I}(a b \mid \lambda) P_{C D}^{I I}(c d \mid \lambda)$. Thus conditioned on $\lambda$ the probabilities to obtain outcome pairs $a, b$ and $c, d$ when measuring $A, B$ and $C, D$ are statistically independent. 
we will provide an entangled state of the four-partite quantum system $\mathfrak{O}$ and specific sets of bi-partite observables each performed by parties $I$ and $I I$ (that each have a bi-partite system) with the following property: These observables give rise to correlations (i.e., joint probability distributions) in the bi-partite subsystems, which violate the Bell inequality in terms of correlations of the previous section (see Eq. (4) ). However, before doing that we have to give the quantum mechanical version of this Bell inequality. We proceed as follows.

Firstly, by a quantum correlation we have in mind the joint probability distribution of two (or more) self-adjoint operators, where each such operator corresponds to an observable of a subsystem. Note that in case of independent observables this equals the distribution of the product of the observables. Such joint probability distributions are uniquely determined by mean values of (sums of) products of projection operators onto linear subspaces. In the remainder we can therefore take quantum correlations to be the joint probability distributions $P_{\hat{Q} \otimes \hat{R}}(q r \mid W)$ that are obtained from products of projection operators $\hat{Q}$ and $\hat{R}$ (with possible outcomes $q, r \in\{0,1\}$ ) for the bi-partite state (density operator) $W$. Of course $\hat{Q}$ and $\hat{R}$ must commute in order for the joint probability distribution to be well defined, but this is ensured since both operators are defined for different subsystems (with each their own Hilbert space) and are therefore commuting.

Secondly, we choose the set of hidden variables to be the set of possible states on the Hilbert space $\mathcal{H}^{\mathfrak{O}}$ of the four-partite system $\mathfrak{O}$, i.e., $\rho(\lambda)=\delta\left(\lambda-W_{0}\right)$, where $W_{0}$ is a quantum state (i.e., density operator) on $\mathcal{H}^{\mathfrak{O}}$. This is possible because quantum mechanics can be regarded as a stochastic hidden variables theory where the hidden variable is the quantum state. Upon assuming local realism, we then recover the same factorisability condition of Eq. (2)

$$
P_{\hat{A} \hat{B}, \hat{C} \hat{D}}\left(a b, c d \mid W_{0}\right)=P_{\hat{A} \hat{B}}^{I}\left(a b \mid W_{I}\right) P_{\hat{C} \hat{D}}^{I I}\left(c d \mid W_{I I}\right),
$$

where $P_{\hat{A} \hat{B}}^{I}\left(a b \mid W_{I}\right)$ is the quantum mechanical joint probability distribution to obtain outcomes $a$ an $b$ when measuring observables $\hat{A}$ and $\hat{B}$ (each associated to a different subsystem) on the bi-partite reduced state $W_{I}$, and the same holds for the other joint probability distribution in the case of party $I I$. This factorisability condition finally gives us the quantum mechanical version of the Bell inequality of Eq. (4) encoding local realism for correlations:

$$
\begin{aligned}
& \left|E_{W_{0}}(\mathfrak{B})\right|=\mid E_{W_{0}}(\hat{A} \hat{B}, \hat{C} \hat{D})+E_{W_{0}}\left(\hat{A} \hat{B},(\hat{C} \hat{D})^{\prime}\right) \\
& +E_{W_{0}}\left((\hat{A} \hat{B})^{\prime}, \hat{C} \hat{D}\right)-E_{W_{0}}\left((\hat{A} \hat{B})^{\prime},(\hat{C} \hat{D})^{\prime}\right) \mid \leq 2,
\end{aligned}
$$

where $E_{W_{0}}(\hat{A} \hat{B}, \hat{C} \hat{D})=\operatorname{Tr}\left[\mathrm{W}_{0} \hat{\mathrm{A}} \otimes \hat{\mathrm{B}} \otimes \hat{\mathrm{C}} \otimes \hat{\mathrm{D}}\right]$, and $\mathfrak{B}$ is the so called Bell operator.

Now that we have the quantum version of the Bell-inequality in terms of correlations, we will provide an example of a violation of it. Consider two sets of two dichotomic observables represented by self-adjoint operators $\hat{a}, \hat{a}^{\prime}$ and $\hat{b}, \hat{b}^{\prime}$ for party $I$ and $I I$ respectively. Each observable acts on the subspace $\mathcal{H}=\mathbb{C}^{2} \otimes \mathbb{C}^{2}$ of the bi-partite system held by the respective party $I$ or $I I$. These observables are chosen to be dichotomous, i.e. to have possible outcomes in $\{-1,1\}$. They are furthermore chosen to be sums of projection operators and thus give rise to unique joint probability distributions on the set of quantum states. Measuring 
these observables thus implies determining some quantum correlations. For these observables $\hat{a}, \hat{a}^{\prime}, \hat{b}$ and $\hat{b}^{\prime}$ the Bell operator $\mathfrak{B}$ on $\mathcal{H}=\mathbb{C}^{2} \otimes \mathbb{C}^{2} \otimes \mathbb{C}^{2} \otimes \mathbb{C}^{2}$ becomes $\mathfrak{B}=\hat{a} \otimes \hat{b}+\hat{a} \otimes \hat{b}^{\prime}+\hat{a}^{\prime} \otimes \hat{b}-\hat{a}^{\prime} \otimes \hat{b}^{\prime}$. The observables have the following form. Firstly,

$$
\hat{a}=\hat{P}_{\psi^{+}}+\hat{P}_{\phi^{+}}-\hat{P}_{\psi^{-}}-\hat{P}_{\phi^{-}},
$$

which is a sum of four projections onto the Bell basis $\left|\psi^{ \pm}\right\rangle=1 / \sqrt{2}(|\uparrow \downarrow\rangle \pm|\downarrow \uparrow\rangle)$ and $\left|\phi^{ \pm}\right\rangle=1 / \sqrt{2}(|\uparrow \uparrow\rangle \pm|\downarrow \downarrow\rangle)$. Here the states $|\uparrow\rangle$ and $|\downarrow\rangle$ are the spin-states for "up" and "down" in the $z$-direction of a single particle respectively, and together they form a basis for $\mathcal{H}=\mathbb{C}^{2}$. Secondly,

$$
\hat{a}^{\prime}=\hat{P}_{|\uparrow \uparrow\rangle}+\hat{P}_{|\uparrow \downarrow\rangle}-\hat{P}_{|\downarrow \uparrow\rangle}-\hat{P}_{|\downarrow \downarrow\rangle},
$$

where the projections are onto the product states $|\uparrow \uparrow\rangle,|\uparrow \downarrow\rangle,|\downarrow \uparrow\rangle,|\downarrow \downarrow\rangle$. And finally,

$$
\begin{gathered}
\hat{b}=\hat{P}_{|\uparrow \uparrow\rangle}+\hat{P}_{|b+\rangle}-\hat{P}_{|b-\rangle}-\hat{P}_{|\downarrow \downarrow\rangle}, \\
\hat{b}^{\prime}=\hat{P}_{|\downarrow \downarrow\rangle}+\hat{P}_{\left|b^{\prime}+\right\rangle}-\hat{P}_{\left|b^{\prime}-\right\rangle}-\hat{P}_{|\uparrow \uparrow\rangle},
\end{gathered}
$$

where we have $|b \pm\rangle=C^{ \pm}(|\uparrow \downarrow\rangle+(1 \pm \sqrt{2})|\downarrow \uparrow\rangle)$ and $\left|b^{\prime} \pm\right\rangle=C^{\mp}(|\uparrow \downarrow\rangle+(-1 \pm$ $\sqrt{2})|\downarrow \uparrow\rangle)$, with normalization coefficients $C^{ \pm}=(4 \pm 2 \sqrt{2})^{-1 / 2} 6$.

Consider now the four particle entangled pure state

$$
|\Psi\rangle=\frac{1}{\sqrt{2}}(|\uparrow \downarrow \uparrow \downarrow\rangle-|\downarrow \uparrow \downarrow \uparrow\rangle) .
$$

The mean value of the Bell operator $\mathfrak{B}$ for the above choice of $\hat{a}, \hat{a}^{\prime}, \hat{b}, \hat{b}^{\prime}$ in the state $|\Psi\rangle$ is equal to

$$
|\operatorname{Tr}[\mathfrak{B}|\Psi\rangle\langle\Psi|]|=2 \sqrt{2} .
$$

This gives us a violation of the Bell inequality of Eq. (6) by a factor of $\sqrt{2}$. This violation proves that quantum correlations cannot be considered to be local elements of reality.

The violation is the maximum value because Cirel'son's inequality [12] (i.e., $|\operatorname{Tr}[\mathfrak{B} W]| \leq 2 \sqrt{2}$ for all quantum states $W)$ must hold for all dichotomic observables $\hat{a}, \hat{a}^{\prime}, \hat{b}, \hat{b}^{\prime}$ on $\mathcal{H}=\mathbb{C}^{2} \otimes \mathbb{C}^{2}$ (possible outcomes in $\{-1,1\}$ ). One can easily see this because for $\hat{a}, \hat{a}^{\prime}, \hat{b}, \hat{b}^{\prime}$ we have that $\hat{a}^{2}=\hat{a}^{\prime 2}=\hat{b}^{2}=\hat{b}^{\prime 2}=\mathbb{1}$, and it thus follows that the proof of Landau [13] of Cirel'son's inequality goes through.

\section{ENTANGLEMENT IS NOT ONTOLOGICALLY RO- BUST}

Entanglement is the fact that certain quantum states of a composite system exist that are not convex sums of product state: 7 . It gives rise to a special kind of

${ }^{6}$ This particular choice of observables $\hat{a}, \hat{a}^{\prime}, \hat{b}, \hat{b}^{\prime}$ on $\mathcal{H}=\mathbb{C}^{2} \otimes \mathbb{C}^{2}$ is motivated by a well-known choice of single particle observables that allows for a maximum violation of the original Bell-CHSH inequality when using the singlet state $\left|\phi^{-}\right\rangle=1 / \sqrt{2}(|\uparrow \uparrow\rangle-|\downarrow \downarrow\rangle)$. This choice is $\hat{a}=-\hat{\sigma_{x}}, \hat{a}^{\prime}=\hat{\sigma_{z}}, \hat{b}=$ $1 / \sqrt{2}\left(-\hat{\sigma}_{z}+\hat{\sigma}_{x}\right), \hat{b}^{\prime}=1 / \sqrt{2}\left(\hat{\sigma}_{z}+\hat{\sigma_{x}}\right)$ all on $\mathcal{H}=\mathbb{C}^{2}$. The analogy can be seen by noting that in this latter choice the (unnormalized) eigenvectors of $\hat{a}$ are $|\uparrow\rangle+|\downarrow\rangle,|\uparrow\rangle-|\downarrow\rangle$, of $\hat{a}^{\prime}$ they are $|\uparrow\rangle,|\downarrow\rangle$, of $\hat{b}$ they are $|\uparrow\rangle+(1+\sqrt{2})|\downarrow\rangle,|\uparrow\rangle+(1-\sqrt{2})|\downarrow\rangle$ and finally of $\hat{b}^{\prime}$ they are $|\uparrow\rangle+(-1+\sqrt{2})|\downarrow\rangle,|\uparrow\rangle+(-1-\sqrt{2})|\downarrow\rangle$.

${ }^{7}$ For completeness, a bipartite state $W$ is entangled iff $W \neq \sum_{i} p_{i} W_{i}^{I} \otimes W_{i}^{I I}$, where $W_{i}^{I}$ and $W_{i}^{I I}$ are arbitrary states of the two subsystems and $\forall p_{i}>0, \sum_{i} p_{i}=1$. A state that is not entangled is 
quantum correlations, called non-classical correlations, which in the pure state case allow one to violate a Bell inequality (for mixed states it is not always the case that entanglement implies violation of a Bell inequality [14]). Furthermore, these correlations can also be used to perform exceptional quantum information and computation tasks.

The SSC theorem of section 2 tells us that entanglement can be completely characterised by the above quantum correlations that it gives rise to. Therefore the result of the previous section also applies to entanglement. Then, if one considers the quantum state description to be complete, entanglement cannot be viewed as ontologically robust in the sense of being a local objective property pertaining to some composite system. If one would do so nevertheless, one can construct a composite system that contains as a subsystem the entanglement (i.e. the entangled system) in question and which would allow for a violation of the Bell inequality of Eq. (6). This implies (contra the assumption) that the entanglement cannot be regarded in a local realistic way, which we take to be a necessary condition for ontological robustness.

It is possible that one thinks that the requirement of local realism is too strong a requirement for ontological robustness. However, that one cannot think of entanglement as a property wich has some ontological robustness can already be seen using the following weaker requirement: anything which is ontologically robust can, without interaction, not be mixed away, nor swapped to another object, nor flowed irretrievably away into some environment. Precisely these features are possible in the case of entanglement and thus even the weaker requirement for ontological robustness does not hold.

These features show up at the level of quantum states when considering a quantum system in conjunction with other quantum systems: entanglement can (i) be created in previously uninteracting particles using swapping, (ii) be mixed away and (iii) flow into some environment upon mixing, all without interaction between the subsystems in question. It is this latter point, the fact that no interaction is necessary in these processes, that one cannot think of entanglement as ontologically robust.

To see that the above weaker requirement for ontological robustness of entanglement does not hold consider the following examples of the three above mentioned features.

(i) Consider two maximally entangled pairs (e.g. two singlets) that are created at spacelike separation, where from each pair a particle is emitted such that these two meet and the other particle of each pair is emitted such that they fly away in opposite directions. Conditional on a suitable joint measurement performed on the pair of particles that will meet (a so called Bell-state measurement) the state of the remaining two particles, although they have never previously interacted, will be 'thrown' into a maximally entangled state. The entanglement is swapped [16].

(ii) Equally mixing the two maximally entangled Bell states $\left|\psi^{ \pm}\right\rangle$gives the separable mixed state

$$
W=\frac{1}{2}\left(P_{\left|\psi^{+}\right\rangle}+P_{\left|\psi^{-}\right\rangle}\right)=\frac{1}{2}\left(P_{|\uparrow \downarrow\rangle}+P_{|\downarrow \uparrow\rangle}\right) .
$$

called separable. For the definition of multipartite entanglement see [15]. 
The entanglement is thus mixed away, without any necessary interaction between the subsystems.

(iii) Equally mixing the following two states of three spin $1 / 2$ particles, where particles 2 and 3 are entangled in both states,

$$
\begin{aligned}
|\psi\rangle & =\frac{1}{2}\left(|\uparrow\rangle^{(1)} \otimes\left|\psi^{-}\right\rangle^{(23)}\right) \\
|\phi\rangle & =\frac{1}{2}\left(|\downarrow\rangle^{(1)} \otimes\left|\psi^{+}\right\rangle^{(23)}\right),
\end{aligned}
$$

gives the state

$$
W=\frac{1}{2}\left(P_{\uparrow}^{(1)} \otimes P_{\psi^{-}}^{(23)}+P_{\downarrow}^{(1)} \otimes P_{\psi^{+}}^{(23)}\right) .
$$

This three-particle state is two-particles entangled although it has no two particle subsystem whose (reduced) state is entangled [15]. The bipartite entanglement has thus irretrievably flowed into the three particle state, again without any necessary interaction between the subsystems.

Does this lack of ontological robustness of entanglement question the popular idea of entanglement as a resource for quantum information and computation tasks? We think it does not. Quantum information theory is precisely a theory devised to deal with the surprising characteristics of entanglement such as the ontologically non-robustness here advocated (and many other features, such as for example teleportation). Entanglement is taken to a be specific type of correlation that can be used as a resource for encoding and manipulating (quantum) bits of information. For that purpose the ontological status of the information or of that which bears the information does not matter. The only thing that matters is that entanglement can be manipulated according to certain rules that allow for interesting information-theoretic and computational tasks.

To conclude this section we should mention that Timpson and Brown [17] do argue for the ontological robustness of entanglement in the mixing case (ii) above by introducing ontological relevance to the preparation procedure of a quantum state, which supposedly can always be captured in the full quantum mechanical description. They introduce the dictinction between 'improper' and 'proper separability', which is analogous to the well known distinction between proper and improper mixtures, to argue that one can retain an ontologically robust notion of entanglement. They thus call the separable mixed state of Eq. (13) improperly separable because the entanglement in the mixture becomes hidden on mixing (i.e, it disappears), although there are some extra facts of the matter that tell that the separable state is in fact composed out of an ensemble of entangled states. We agree that "there need be no mystery at the conceptual level over the disappearance" (sec 2. in ref. [17]), and the introduced distinction between proper and improper separability indeed shows this. However, we are not convinced that their analysis of the improperly separable states allows for ontological robustness of entanglement.

Timpson and Brown argue that using the extra facts of the matter an observer is able to perform a place selection procedure that would allow the ensemble to be separated out into the original statistically distinct sub-ensembles [i.e., into the entangled states]. According to them it is always the case that such a procedure 
exists in principle: "all that is required is access to these further facts" (sec. 1 in ref. [17]).

However, we believe that it is very well conceivable that according to quantum mechanics we do not in principle always have access to these extra facts. Perhaps the interactions between the object systems involved in the preparation procedure and the environment are such that the observer cannot become correlated to both the extra facts and the objects states in the right way for the facts to be accessable, or the interactions could be such that no classical record of the extra facts could possibly be left in the environment. To put it differently, although we agree that in the case of improper separability one can uphold an ignorence interpretation of the state in question and that furthermore the ignorance is in principle about some extra facts of the matter, we do not agree that it is certain that the ignorance about these extra facts of the matter can be removed by the observer in accordance with the dynamics of quantum mechanics in all conceivable preparation procedures. The issue thus awaits a (dis)proof of principle, which the author wants to undertake in the near future.

\section{DISCUSSION AND CONCLUSION}

The Bell inequality violation of section 4 tells us that despite the fact that a quantum state of a composite system is determined by the correlations between each of its possible subsystems, one cannot conclude that they are determined (by the quantum state) in a local way. Just like values of quantities correlations cannot be used to build up a world consisting out of some local realistic structure. We have that mathematically quantum correlations determine the quantum state, but ontologically they cannot be considered to be atomic building blocks of the world. Furthermore, although entanglement is taken to be a resource in quantum information theory, we have argued that it cannot be considered ontologically robust because it is not a local objective property and furthermore that without interaction it can be mixed away, swapped to another object, and flowed irretrievably into some environment.

The Bell inequality argument of section 4 was inspired by the work of Cabello 3] and Jordan [4] who obtain almost exactly the same conclusion, although by different arguments. The argument of Cabello differs the most from ours because he uses a different conception of what a quantum correlation is. His argument speaks of types of correlations which are associated with eigenvalues of product observables. We believe this notion to be less general than our notion of quantum correlation which only takes joint probability distributions to be correlations. Jordan's argument, in contrast, does in effect use the same notion of quantum correlation as we do. He considers mean values of products of observables and since these are determined by mean values of (sums of) products of projection operators he restricts himself to the latter. He thus uses the same notion as we do because the latter determine all joint probability distributions.

However, Cabello and Jordan both need perfect correlations for their argument to work because the state dependent GHZ- or Hardy argument they use (Cabello uses both, Jordan only the latter argument) need such strong correlations. Our Bell inequality argument does not rely on this specific type of correlation because 
statistical correlations already suffice to violate the Bell inequality here presented. We therefore believe our argument has an advantage over the one used by Cabello and Jordan, because it is more amenable to experimental implementation.

In fact, the Bell-inequality argument here presented can be readily implemented using current experimental technology. Indeed, it is already possible to create fully four-particle-entangled states [18] and measurement of the four observables $\hat{a}, \hat{a}^{\prime}, \hat{b}, \hat{b}^{\prime}$ of Eq.(7)-(10) seems not to be problematic since they are sums of ordinary projections. Furthermore, as said before, there is no need to produce perfect correlations; statistical correlations will suffice. We therefore hope that in the near future experiments testing our argument will be carried out.

Lastly, returning to the questions stated in the introduction, in so far as Mermin in his [2] is committed to take correlations (as we have defined them here) to be locally real (which we think he is), his tentative interpretation is at odds with predictions of quantum mechanics and would allow, in view of the argument given here, for an experimental verdict.

\section{Acknowledgements}

I would like to thank Jos Uffink for valuable discussions, Sven Aerts and N. David Mermin for helpful comments on an earlier draft and Harvey Brown for stimulating comments on this topic, even though the latter go back many years.

\section{References}

[1] J.S. Bell, " On the Einstein-Podolsky-Rosen paradox," Physics 1, 195 (1964).

[2] N.D. Mermin, in a series of papers, tried to defend this fundamental interpretational difference between values of quantities and correlations. N.D. Mermin, "What is quantum mechanics trying to tell us?," Am. J. Phys. 66, 753 (1998); N.D. Mermin, "What do these correlations know about reality? Nonlocality and the absurd," Found. Phys. 29, 571 (1999); N.D. Mermin, "The Ithaca interpretation of quantum mechanics," Pramana 51, 549 (1998).

[3] A. Cabello, "Quantum correlations are not contained in the initial state," Phys. Rev. A 60, 877 (1999).

[4] T.F. Jordan, "Quantum correlations violate Einstein-Podolsky-Rosen assumptions," Phys. Rev. A 60, 2726 (1999).

[5] S. Kochen and E.P. Specker, "The problem of hidden variables in quantum mechanics," J. Math. Mech. 17, 59 (1967).

[6] D.M. Greenberger, M.A.Horne and A. Zeilinger, "Going beyond Bell's theorem," in Bell's theorem, quantum theory and conceptions of the universe, M. Kafatos (Ed.), (Kluwer Academic, Dordrecht, 1989), p. 69; D.M. Greenberger, M.A.Horne, A. Shimony, and A. Zeilinger, "Bell's theorem without inequalities," Am. J. Phys. 58, 1131 (1990).

[7] L. Hardy, "Nonlocality for two particles without inequalities for almost all states," Phys. Rev. Lett. 71, 1665 (1993). 
[8] J. Barrett, "Information processing in non-signalling theories," Los Alamos e-print archive, http://arxiv.org/abs/quant-ph/0508211.

[9] W.K. Wootters,"Local accessibility of quantum states," in Complexity, Entropy and the Physics of Information, W.H. Zurek (Ed.), (Addison-Wesley, Boston, 1990), p. 39.

[10] See the first paper by N.D. Mermin in [2].

[11] J.F. Clauser, W.A. Horne, A. Shimony, and R.A. Holt, "Proposed experiment to test local hidden-variable theories," Phys. Rev. Lett. 23, 880 (1969).

[12] B.S. Cirelson, "Quantum generalizations of Bell's inequality," Lett. Math. Phys. 4, 93 (1980).

[13] L.J. Landau, "On the violation of Bell's inequality in quantum theory," Phys. Lett. A. 120, 54 (1987).

[14] R.F. Werner, "Quantum states with Einstein-Podolsky-Rosen correlations admitting a hidden-variable model," Phys. Rev. A 40, 4277 (1989).

[15] M. Seevinck and J. Uffink, "Sufficient conditions for three-particle entanglement and their tests in recent experiments," Phys. Rev. A 65, 012107 (2001).

[16] M. Żukowski, A. Zeilinger, M.A. Horne and A.K. Ekert, "'Event-readydetectors' Bell experiment via entanglement swapping," Phys. Rev. Lett. 71, 4287 (1993).

[17] C.G. Timpson, H.R. Brown, "Proper and improper separability," Int. J. Quant. Inf. 3, 679 (2005).

[18] C.A. Sackett, et al., "Experimental entanglement of four particles," Nature 404, 256 (2000); Z. Zhao, et al., "Experimental violation of local realism by four-photon Greenberger-Horne-Zeilinger entanglement," Phys. Rev. Lett. 91, 180401 (2003); Z. Zhao, et al., "Experimental demonstration of fivephoton entanglement and open-destination teleportation," Nature (London) 430, 54 (2004); M. Eibl, et al., "Experimental observation of four-photon entanglement from parametric down-conversion," Phys. Rev. Lett. 90, 200403 (2003). 